\title{
Obligatorische Versicherung gegen Schäden infolge von Naturkatastrophen
}

\author{
Wie schon die Hochwasserereignisse 2002 und 2013 haben auch die Folgen der \\ Flutkatastrophe im Juli 2021 dramatisch die Notwendigkeit von gesellschaftlichen \\ Klimaanpassungsstrategien in Deutschland aufgezeigt. Bund und Länder haben zum dritten \\ Mal eine Arbeitsgruppe mit der verfassungsrechtlichen Prüfung einer Versicherungspflicht \\ gegen Elementarschäden beauftragt. Während sich die grundsätzlichen Argumente \\ in der Debatte um die Einführung wiederholen, lohnt sich ein Blick auf die zentralen \\ Ausgestaltungsfragen einer potenziellen Versicherungspflicht. Die häufig vorgebrachten \\ Kritikpunkte gegen eine Versicherungspflicht legen konkrete Ausgestaltungsannahmen \\ zugrunde und sprechen daher nicht pauschal gegen das Instrument.
}

Die finanziellen Schäden, die durch Naturkatastrophen verursacht werden, sind enorm. Im Fall der jüngsten Flutkatastrophe werden sie auf 29,2 Mrd. Euro geschätzt und übersteigen damit um ein Mehrfaches die Schäden früherer Flutkatastrophen (Tageschau, 2021). In einer aktuellen Studie wird der Klimawandel für die Flutkatastrophe im Juli 2021 mitverantwortlich gemacht (Pfeiffer, 2021). Auch im öffentlichen Diskurs bestehen kaum Zweifel daran, dass ein Zusammenhang zwischen dem globalen Temperaturanstieg und zunehmenden und stärkeren Naturereignissen besteht. Der Gesamtverband der Deutschen Versicherungswirtschaft (GDV) prognostiziert für die nächsten Jahre einen Anstieg von Schäden durch Naturkatastrophen. Abhängig vom zugrunde liegenden Klimamodell werden sich die Schäden durch Hochwasser demnach bis 2100 verdoppeln oder sogar verdreifachen (GDV, 2019).

Laut dem GDV sind in Deutschland insgesamt weniger als die Hälfte aller Wohngebäude gegen Elementarschäden versichert (GDV, 2021a). Bund und Länder stellen in Krisensituationen regelmäßig schnell und unbürokratisch hohe staatliche Nothilfegelder bereit. Es steht außer Frage, dass die staatlichen Hilfsgelder bei vergangenen Katastrophen richtig und notwendig waren. Allerdings werden bei der Zuteilung, Organisation und Verwaltung der Ad-hoc-Zahlungen Effizienzverluste in Kauf genommen, die durch eine gut durchdachte, langfristige gesellschaftliche Vorsorgestrategie vermieden werden könnten. Zudem steckt hinter den staatlichen Ad-hoc-Hilfen die Gefahr der politischen Willkür. Faktoren wie die mediale Auf-

(C) Der/die Autor:in 2022. Open Access: Dieser Artikel wird unter der Creative Commons Namensnennung 4.0 International Lizenz veröffentlicht (creativecommons.org/licenses/by/4.0/deed.de).

Open Access wird durch die ZBW - Leibniz-Informationszentrum Wirtschaft gefördert. merksamkeit, die auf die Naturkatastrophe gerichtet wird, die Haushaltslage oder der nächste Wahltermin können die Höhe der Hilfsgelder stark beeinflussen. ${ }^{1}$

Um diesen Problemen entgegenzuwirken, wird aktuell die Einführung einer obligatorischen Elementarschadenversicherung diskutiert, wie es sie bereits in anderen europäischen Ländern gibt. Auf Drängen einer Sonderumweltministerkonferenz im Oktober sowie mehrerer Ministerpräsident:innen wurde auf der Justizministerkonferenz im November eine Arbeitsgruppe gegründet, welche die Zulässigkeit einer Versicherungspflicht gegen Elementarschäden verfassungsrechtlich prüfen soll. Die Ergebnisse sollen im Frühjahr vorliegen. Es ist nicht das erste Mal, dass diese Diskussion in Deutschland geführt wird. Bereits nach den Flutkatastrophen 2002 und 2013 wurden Bund-Länder-Arbeitsgruppen geschaffen, die eine Pflicht zur Versicherung gegen Elementarschäden diskutieren und gangbare Wege aufzeigen sollten. Beide Arbeitsgruppen haben sich im Ergebnis gegen eine obligatorische Elementarschadenversicherung ausgesprochen. Während sich zentrale Abwägungen über die Jahre wiederholen, könnten sie angesichts der enormen

1 In den USA konnte z. B. empirisch gezeigt werden, dass die Höhe von in der Vergangenheit gezahlten Katastrophenhilfen stark davon abhängt, ob gerade ein Wahljahr war und ob die betroffene Region wahlstrategisch wichtig für den Präsidenten war (Garrett und Sobel, 2003).

Ann-Kristin Becker und Christoph Oslislo sind wissenschaftliche Mitarbeitende am Institut für Wirtschaftspolitik an der Universität zu Köln. 
Schäden der jüngsten Flutkatastrophe und angepassten Klimaprognosen neu gewichtet werden. Daher lohnt sich heute ein Blick auf die zentralen Fragen zur Ausgestaltung einer potenziellen obligatorischen Versicherung gegen Elementarschäden. Wer soll die Versicherung anbieten: eine staatliche Monopolversicherung oder mehrere private Anbieter? Und wie sollen die Prämien gestaltet werden: risikodifferenziert oder zu einem Einheitstarif?

\section{Geringe Absicherung gegen Elementarschäden}

\section{Elementarschadenversicherung}

In Deutschland gibt es einen privaten Versicherungsmarkt, auf dem sich Haushalte gegen Naturgefahren absichern können. Die Elementarschadenversicherung ist in Deutschland eine Zusatzpolice, die zur Wohngebäudeversicherung hinzugebucht werden kann. Die Prämienhöhe in der Elementarschadenversicherung für Wohngebäude ist aktuell vom individuellen Naturgefahrenrisiko des Wohngebäudes abhängig. Um diese Risiken einzuschätzen, haben die deutschen Versicherer ein Zonierungssystem für Überschwemmung, Rückstau und Starkregen (ZÜRS GEO) eingerichtet, das die Gebäude in Deutschland in vier Gefährdungsklassen (GK) unterteilt. Der Großteil aller Gebäude in Deutschland (92,4\%) liegt in der GK 1 und gilt damit als nicht von Hochwasserrisiken betroffen. Die GK 2 umfasst $6,1 \%$ aller Gebäude. In dieser Gefährdungsklasse wird das Hochwasserrisiko auf seltener als einmal in 100 Jahren geschätzt. 1,1\% der Gebäude liegen in der GK 3, mit einem Hochwasserrisiko von einmal in 10 bis 100 Jahren. Die restlichen 0,4\% der Gebäude liegen in einem Gebiet mit einem Hochwasserrisiko von einmal in zehn Jahren (GDV, 2021b).

\section{Ursachen für geringe Versicherungsdichte}

Oftmals werden angebotsseitige Aspekte für die geringe Versicherungsdichte verantwortlich gemacht. Damit die Elementarschadenversicherung für Wohngebäude in Hochrisikogebieten für Versicherer tragbar ist, müssten die Versicherungsprämien teilweise sehr hoch sein. Aus Sorge vor einer Rufschädigung könnten Versicherungen Risikoselektion betreiben und einzelnen Hauseigentümer:innen kein Versicherungsangebot machen. ${ }^{2}$ Außerdem ist denkbar, dass Anbieter in bestimmten Fällen die Kosten einer Angebotserstellung gar nicht erst auf sich nehmen, da sie davon ausgehen, dass das Angebot von den Hauseigentümer:innen sowieso abgelehnt wird. Häuser im Bestand in Hochrisikogebieten werden in diesem Fall als

2 Konkret könnten Versicherer Sorge davor haben, dass Hauseigentümer:innen aufgrund eines unzumutbaren Versicherungsangebots andere Versicherungen bei dem Anbieter kündigen bzw. keine weiteren Angebote zu anderen Versicherungen einholen. unversicherbare Risiken deklariert. Über das Ausmaß der angebotsseitigen Probleme finden sich widersprüchliche Aussagen. Während der GDV angibt, dass $99 \%$ aller Immobilien in Deutschland unter zumutbaren Bedingungen versicherbar sind, zweifelt die Verbraucherzentrale Rheinland-Pfalz diese Zahl im Rahmen einer Studie an (GDV, 2021c; Verbraucherzentrale Rheinland-Pfalz, 2018).

Nachfrageseitig gibt es drei theoretische Argumente, die eine geringe Versicherungsdichte erklären können. Zunächst kann es sein, dass es einem großen Anteil der Hauseigentümer:innen gar nicht bewusst ist, dass ihr Gebäude nicht gegen bestimmte Naturkatastrophen versichert ist (GDV, 2021a). Da Schäden durch Stürme durch die Wohngebäudeversicherung abgedeckt sind, könnten Verbraucher:innen beispielsweise davon ausgehen, dass auch Schäden durch andere Naturgefahren abgesichert sind. Bei Neuabschlüssen wird heute allerdings in der Regel darauf hingewiesen, dass Elementarschäden nicht automatisch in der Wohngebäudeversicherung enthalten sind und eine zusätzliche Police abschlossen werden muss. Zudem gibt es seit längerem ein breites, leicht zugängliches Informationsangebot über Naturgefahren und Präventionsmaßnahmen (Gross, Schwarze und Wagner, 2019).

Es gibt Evidenz dafür, dass das eigene Risiko, in Zukunft von einem Extremwetterereignis betroffen zu sein, von vielen unterschätzt wird. Gross, Schwarze und Wagner (2019) erklären mangelnde Awareness unter anderem dadurch, dass sich Menschen bei der Risikobewertung häufig auf Erfahrungswissen verlassen. Das vergleichsweise seltene Auftreten von Naturkatastrophen kann so als Erklärungsansatz für eine systematische Unterschätzung der tatsächlichen Risiken bei Hauseigentümer:innen herangezogen werden.

Das in der Literatur meistdiskutierte Problem auf der Nachfrageseite basiert auf der Annahme, dass Individuen sich darauf verlassen, dass der Staat im Katastrophenfall finanzielle Hilfen für Reparaturen (im Extremfall für den Wiederaufbau) bereitstellt. Die Antizipation von staatlichen Hilfsgeldern kann theoretisch die Anreize verringern, selber eine Versicherung abzuschließen. In der Literatur wird diese Anreizproblematik Charity Hazard genannt. Einige empirische Arbeiten geben Hinweise darauf, dass es tatsächlich einen starken Zusammenhang zwischen der Versicherungsnachfrage und antizipierten Hilfsgeldern gibt (z.B. Andor, Osberghaus und Simora, 2020; Raschky und Weck-Hannemann, 2007; Browne und Hoyt, 2000; Lewis und Nickerson, 1989).

\section{Lösungsvorschläge}

Es ist davon auszugehen, dass die Ursachen der geringen Versicherungsdichte sowohl auf der Angebots- als 
auch auf der Nachfrageseite liegen. Eine unterschiedliche Gewichtung der jeweiligen einzelnen Argumente kann zu sehr unterschiedlichen Positionen hinsichtlich der Frage führen, mit welchen wirtschaftspolitischen Instrumenten die Versicherungsdichte erhöht werden kann.

Wird die Ursache für die geringe Versicherungsdichte hauptsächlich in einem unzureichenden Angebot gesehen, könnte ein einseitiger Versicherungszwang einen effektiven Markteingriff darstellen. In diesem Modell wären die Versicherer dazu verpflichtet, allen Wohngebäudeeigentümer:innen ein Versicherungsangebot zu machen. Allerdings müssten zusätzliche Maßnahmen getroffen werden, die sicherstellen, dass für alle ein Versicherungsangebot zu wirtschaftlich tragbaren Bedingungen zur Verfügung steht und der Kontrahierungszwang nicht durch unzumutbare Versicherungsprämien oder Selbstbehalte umgangen werden kann.

Um auf der Nachfrageseite dem Problem des unzureichenden Risikobewusstseins gegenüber Naturgefahren zu begegnen, ist der naheliegendste Ansatz eine Verbesserung des Informationsstands in der Gesellschaft und eine Schärfung der Awareness für Naturgefahren. Hier könnte zum einen über eine umfangreiche Informationspflicht von der Gebäudeversicherung zum Zeitpunkt des Versicherungsabschlusses nachgedacht werden. Zum anderen kann gehofft werden, dass die jüngste Flutkatastrophe und die aktuelle Debatte zu einer zumindest temporären Erhöhung der Awareness führen. ${ }^{3} \mathrm{Ob}$ Informations- und Awareness-Kampagnen das Problem der geringen Versicherungsdichte allerdings allein lösen können, ist fraglich. Eine empirische Untersuchung der Effektivität von groß angelegten Awareness-Kampagnen von Osberghaus und Hinrichs (2021) findet bisher keinen signifikanten Effekt der durchgeführten Kampagnen auf die Versicherungsnachfrage deutscher Haushalte.

Vermehrt kommt auch der Vorschlag auf, Nudges einzusetzen, um die Versicherungsnachfrage zu erhöhen. Zu den effektivsten Nudges zählt die Änderung von Standardvorgaben (Defaults). Abgesehen von positiven gesamtgesellschaftlichen Effekten einer höheren Versicherungsdichte führen Defaults insbesondere dann zu Wohlfahrtsgewinnen, wenn davon auszugehen ist, dass ein großer Teil der Individuen sich zwar gern anders verhalten würde, allerdings aufgrund hoher Transaktionskosten im Status quo verharrt. Dieser Nudge findet bereits in der Versicherungswirtschaft Anwendung. So existieren Zeichnungsrichtlinien, die Versicherungen dazu anhalten, bei Neuabschlüssen von Wohn-

3 Tatsächlich verzeichnen Versicherungen nach der Flutkatastrophe zumindest kurzfristig ein deutlich höheres Interesse an Elementarschadenversicherungen (dpa, 2021). Ob die verheerenden Ereignisse dieses Mal dazu führen, dass das Thema im „sozialen Langzeitgedächtnis“ (Welzer, 2008) verankert wird, bleibt abzuwarten. gebäudeversicherungen Elementarschadenversicherungen mit anzubieten (Gross, Schwarze und Wagner, 2019). Eine Studie der Verbraucherzentrale Rheinland-Pfalz (2018) legt allerdings nahe, dass Versicherungen, insbesondere bei Gebäuden in Hochrisikogebieten, häufig von dieser Richtlinie abweichen. Hier könnte darüber nachgedacht werden, die Zeichnungsrichtlinie zu einer obligatorischen Opt-OutLösung auszubauen. Allerdings bleibt fraglich, ob Transaktionskosten die geringe Versicherungsdichte erklären können, da davon auszugehen ist, dass sich der Mehraufwand zum Abschluss einer zusätzlichen Police zeitgleich mit dem Abschluss einer Wohngebäudeversicherung in Grenzen hält. Ein Default-Nudge kann aber dennoch wirksam sein. Beispielsweise könnten Standardvorgaben als Empfehlung oder Hinweis auf eine Norm verstanden werden oder überhaupt erst auf das Thema aufmerksam machen und auf diese Weise die Versicherungsdichte erhöhen.

Alle drei Vorschläge - Kontrahierungszwang, Informationsund Awarenesskampagnen und Nudges - zielen hauptsächlich auf Neuabschlüsse ab und adressieren nicht das Problem des Charity Hazards, das von vielen als zentrales Argument zur Erklärung der geringen Versicherungsdichte genannt wird. Um diesem Problem entgegenzuwirken, wurde 2017 auf der Umweltministerkonferenz beschlossen, dass staatliche Hilfen nur noch dann gezahlt werden, wenn festgestellt werden kann, dass sich Betroffene erfolglos um eine Versicherung bemüht haben oder die Bedingungen für die angebotene Versicherung wirtschaftlich unzumutbar waren (BMU, 2017). Einige Bundesländer wie Bayern oder Nordrhein-Westfalen gingen sogar noch weiter und legten fest, dass Nothilfen nur noch in wenigen Härtefällen gewehrt werden (GDV, 2019). Bei der jüngsten Flutkatastrophe im Juli 2021 hat sich allerdings gezeigt, dass bei Katastrophen mit einer großen medialen Aufmerksamkeit politisch nur schwer an diesen Ankündigungen festgehalten werden kann. Bund und Länder haben entgegen ihren vorherigen Beschlüssen wieder schnelle und unbürokratische Sofort- und Aufbauhilfe für Betroffene bereitgestellt. Auf einen Nachweis unversicherbarer Risiken wurde größtenteils verzichtet. Ungeachtet dessen kann angezweifelt werden, ob die Verweigerung staatlicher Unterstützung im Katastrophenfall in einem Sozialstaat gesellschaftlich erwünscht wäre. ${ }^{4}$

Sieht man erstens die Ursache der geringen Abdeckung in den meisten Bundesländern zumindest auch auf der Nachfrageseite, glaubt man zweitens, dass die Fehlanreize aufgrund des Charity Hazards eine entscheidende Ursache für die geringe Versicherungsdichte darstellen und will man

4 Ein Vergleich mit anderen europäischen Ländern zeigt, dass es ein reines Marktmodell in keinem Land gibt. Unregulierte Versicherungsmärkte sind immer mit staatlichen Hilfen im Katastrophenfall verbunden (Schwarze, 2019). 
drittens daran festhalten, Betroffene mit den zum Teil existenzbedrohenden finanziellen Folgen von Naturkatastrophen nicht alleine zu lassen, drängt sich die Idee einer verpflichtenden Naturgefahrenabsicherung für alle Wohngebäude nahezu auf. ${ }^{5}$ Dieses Instrument stellt einen starken Eingriff in die Privatautonomie und Vertragsfreiheit dar. Die zusätzlichen Kosten würden nicht nur Hauseigentümer:innen treffen, sondern alle Haushalte in Deutschland, weil die Versicherungsprämien gemäß der Betriebskostenverordnung von Wohnungseigentümer:innen auf Mietende umgelegt werden können. Selbst wenn dies anders gestaltet werden sollte, würde das Mietniveau mittelfristig ansteigen und die Kosten dadurch indirekt auf die Mietenden umgelegt werden. Trotz dieses erheblichen Eingriffs werden die Stimmen lauter, die eine verpflichtende Elementarschadenversicherung vor dem Hintergrund immer häufiger auftretender Extremwetterereignisse mittlerweile als verhältnismäßig ansehen.

\section{Ausgestaltung einer obligatorischen Versicherung}

Die Idee einer Versicherungspflicht zur Schaffung von Solidargemeinschaften und zur Vermeidung von Risikoselektion ist nicht neu. In Deutschland basieren z.B. große Teile der Sozialversicherung (Arbeitslosen-, Renten-, Kranken-, Unfall- und Pflegeversicherung) auf einer Versicherungspflicht. ${ }^{6}$ Auch eine Pflichtversicherung gegen Elementarschäden gab es bereits bis 1993 in Baden-Württemberg. ${ }^{7}$ Während immer mehr Politiker:innen mit dem Vorschlag sympathisieren und auch viele Ökonom:innen eine obligatorische Versicherung gegen Elementarschäden befürworten, gehen bei der genauen Gestaltung die Meinungen stark auseinander.

\section{Versicherungspflicht oder Pflichtversicherung?}

Eine Kernfrage bei der Ausgestaltung einer Versicherungspflicht für Elementarschäden ist, wer Hauseigentümer:innen die Versicherung anbieten soll. Hier werden zwei Modelle

5 Ein anderer Ansatz, um dem Problem der antizipierten Hilfsgelder zu begegnen, wäre die Vorgabe, dass die Wohngebäudeversicherung, die in der Regel von Banken bei der Finanzierung des Hauses gefordert wird, nur noch im Paket mit einer Elementarschadenversicherung angeboten werden darf. Allerdings könnte das Problem eines unzureichenden Versicherungsangebots durch diese Lösung sogar verschärft werden, wenn Versicherer auf die Bündelungspflicht reagieren, indem sie zukünftig in gefährdeten Gebieten nicht einmal mehr Wohngebäudeversicherungen anbieten. Würde daraus folgen, dass die Maßnahme mit einem Kontrahierungszwang gekoppelt wird, kann argumentiert werden, dass es sich bei dieser Lösung um eine Art „Versicherungspflicht durch die Hintertür" handeln würde, der eine direkte und transparente Lösung vorzuziehen wäre.

6 Auch einige Versicherungen, bei denen es vorrangig um den Schutz Dritter geht, sind in Deutschland verpflichtend, beispielsweise die Kfz-Haftpflicht- und die Berufshaftpflichtversicherung.

7 Viele Hauseigentümer:innen haben ihre Elementarschadenversicherung nach Abschaffung der Versicherungspflicht bis heute nicht gekündigt, wodurch die Versicherungsdichte in diesem Bundesland heute überdurchschnittlich hoch ist (94\%). Zum Vergleich: In Bremen beträgt die Versicherungsdichte $23 \%$ (GDV, 2021a). diskutiert: eine Pflichtversicherung und eine Versicherungspflicht. Im ersten Fall werden die Eigentümer:innen dazu verpflichtet, einen Versicherungsvertrag mit einem öffentlich-rechtlichen Versicherungsträger abzuschließen, bei dem es sich um einen (regionalen) Monopolversicherer handelt. Im zweiten Fall werden alle Betroffenen dazu angehalten, eine Elementarschadenversicherung abzuschlieBen, wobei sie zwischen verschiedenen privaten Versicherungsanbietern wählen können. Zur Sicherstellung eines entsprechenden Versicherungsangebots ist eine Versicherungspflicht in der Regel mit einem Kontrahierungszwang für die Versicherungsanbieter verbunden.

Aus ökonomischer Perspektive sprechen für eine staatliche Monopolversicherung insbesondere zwei theoretische Argumente: Erstens können Versicherungsnehmer in diesem Fall nicht zu Wettbewerbern abwandern, wodurch die Versicherung sich Werbe-, Vertreter- und Akquisitionskosten sparen kann. Durch die Betriebskostenersparnis kann ein Monopolist die Versicherung theoretisch zu einem niedrigeren Prämienniveau anbieten als Versicherer in einem Wettbewerbsmarkt. Dies wird dadurch verstärkt, dass staatliche Monopolversicherer keine Gewinne ausschütten. Außerdem sinken auf Seiten der Nachfrager Informationssuchkosten, da sie keine Angebote verschiedener Anbieter vergleichen müssen. Zweitens kann argumentiert werden, dass Monopolversicherer maßgeblich zur Prävention beitragen, da sie mehr Anreize für Investitionen in Präventionsmaßnahmen haben und sich aktiver in der kommunalen Vorsorgeplanung einbringen können (Schwarze, 2019). Zunächst könnte man davon ausgehen, dass auch private Versicherer Anreize haben, Innovationen bei der Schadensvermeidung voranzutreiben und über Interessenverbände Einfluss auf politische Entscheidungen zu nehmen. Allerdings gibt es auf Wettbewerbsmärkten ein Trittbrettfahrerproblem, da alle Wettbewerber von bestimmten lokalen Präventionsmaßnahmen profitieren würden, unabhängig davon, ob sie sich an der Finanzierung bzw. an den Kosten zur Durchsetzung der Entscheidungen beteiligen.

Bei einer staatlichen Monopolversicherung muss jedoch beachtet werden, dass die dargestellte Anreizkompatibilität in der Praxis nicht selbstverständlich ist, wie zentrale Modellansätze der Ökonomischen Theorie der Bürokratie zeigen. So sollte z.B. sichergestellt werden, dass die Anreize für die neue Institution so gestaltet sind, dass die Versicherung nicht reine Budget- und Output-Maximierung im Vordergrund stellt oder Versicherungsbeiträge zur Finanzierung sachfremder Projekte instrumentalisiert. Stattdessen sollte ein möglichst effizientes gesellschaftliches Risikomanagement das Ziel sein. Zudem muss bedacht werden, dass Aufbau, Betrieb und Überwachung einer neuen Institution mit dem Auftrag, alle Gebäudeeigentümer:innen in Deutschland zu versichern, mit hohen Kosten verbunden wäre - Kosten, die vor dem Hin- 
tergrund eines etablierten privaten Versicherungsmarkts in Deutschland möglicherweise vermeidbar wären.

Demgegenüber stehen die grundsätzlichen Vorteile, die Ökonom:innen typischerweise in dezentralen Wettbewerbslösungen sehen. Diese sind insbesondere dynamische Innovationsanreize und Anreize zur effizienten Betriebsorganisation. Auf wettbewerblichen Versicherungsmärkten müsste die individuelle Risikoeinschätzung sowie ein sinnvoller Präventionsmaßnahmenkatalog nicht von Bürokrat:innen festgelegt werden. Damit können Prozesse möglicherweise anreizkompatibler und effizienter gestalten werden. Privater Wettbewerb kann zudem für eine Angebotsvielfalt sorgen, die bei einem Monopolversicherer nicht gegeben ist. Es kann z. B. eine Angebotsdifferenzierung hinsichtlich des Kundenservices oder diverser anderer Zusatzleistungen erfolgen, die zu einem insgesamt präferenzgerechteren Angebot führen können.

In der Schweiz sind interessanterweise beide Modelle vorzufinden: In den meisten Kantonen sind Gebäudeeigentümer:innen verpflichtet, ihre Gebäude gegen Elementarschäden bei der kantonalen Monopolversicherung zu versichern. In drei Kantonen besteht zwar ebenfalls eine Versicherungspflicht für Gebäudeeigentümer:innen - allerdings können diese zwischen verschiedenen privaten Versicherungsanbietern wählen, die im Wettbewerb zueinander stehen. Es zeigt sich, dass die Versicherungsprämie, die von Monopolversicherern verlangt wird, deutlich geringer ist, als jene die von privaten Versicherungsanbietern veranschlagt wird. Außerdem investieren die kantonalen Monopolisten einen erheblichen Anteil ihrer Prämieneinkünfte in Präventionsmaßnahmen und wirken in kantonalen Entscheidungsgremien zur öffentlichen Raum- und Katastrophenschutzplanung mit. Ihre Schadensbelastung liegt, im Vergleich zu Kantonen mit privaten Versicherungsanbietern, deutlich niedriger (von Ungern-Sternberg, 2002). Allerdings unterscheidet sich der institutionelle Rahmen in der Schweiz aufgrund der kantonalen Strukturen stark von Deutschland und ein Vergleich sollte mit Vorsicht betrachtet werden. Zudem existieren die kantonalen Gebäudeversicherer teilweise schon seit dem 19. Jahrhundert, wohingegen sich in Deutschland über die Jahre ein privater Versicherungsmarkt für Wohngebäudeund Elementarschadenversicherungen etabliert hat, dessen Strukturen und Know-how genutzt werden sollten.

Abwägungen zwischen Äquivalenz und Solidarität

Ein weiteres Streitthema bei der Ausgestaltung einer obligatorischen Elementarschadenversicherung dreht sich um die Frage, wie die Prämien gestaltet werden sollten. Auf der einen Seite steht der Vorschlag der Schaffung einer Solidargemeinschaft mit einheitlichen Tarifen für alle
Hauseigentümer:innen (z.B. in Abhängigkeit vom Gebäudewert). Im Kern dieser Idee liegt die Vorstellung, dass nur auf diese Weise bezahlbare bzw. zumutbare Versicherungsprämien für alle Hauseigentümer:innen sichergestellt werden können. Zudem lässt sich ins Feld führen, dass die zunehmende Gefährdung durch Extremwetterereignisse als Folge des Klimawandels eine direkte Konsequenz gesamtgesellschaftlichen Verhaltens ist. Dem umweltrechtlichen Verursacherprinzip folgend könnte argumentiert werden, dass die gesamte Gesellschaft folglich in eine Solidargemeinschaft treten und diese Kosten gemeinsam stemmen sollte. Allerdings lässt sich damit nur schwer begründen, weshalb auch Neubauten in Hochrisikogebieten zukünftig von der Solidargemeinschaft profitieren sollten.

Die Nachteile einer einheitlichen Versicherungsprämie liegen auf der Hand und wiegen schwer. Der Solidargedanke hinter einheitlichen Prämien bedeutet gleichzeitig, dass hohe Risiken durch niedrige Risiken subventioniert werden. Dies liegt daran, dass für die Berechnung der Prämienhöhe ein durchschnittliches Schadensrisiko angesetzt werden muss, wodurch tendenziell die Prämie für niedrige Risiken zu hoch und für hohe Risiken zu niedrig ist. Wenn sich in Zukunft auch Hauseigentümer:innen in weniger exponierten Lagen zu relativ hohen Prämien versichern müssen, könnte dies in der Gesellschaft als ungerecht empfunden werden. Des Weiteren führt die Umverteilung zugunsten von hochwassergefährdeten Gebäuden dazu, dass Anreize zur privaten Vorsorge im Rahmen von Versicherungsverträgen nur noch über andere Gestaltungsaspekte, z.B. Selbstbehalte, gesetzt werden können. Letztlich wird durch eine Einheitsprämie das Bauen in riskanten Lagen subventioniert (ex ante Moral Hazard).

Auf der anderen Seite steht der Vorschlag, dass das Versicherungssystem, wie bei anderen Versicherungen üblich, auf dem Äquivalenzprinzip aufgebaut wird. Demzufolge sollten Versicherungsbeiträge bei Abschluss eines Vertrages grundsätzlich äquivalent zu den individuellen Risikofaktoren kalkuliert werden. Hierbei müssten sich die Prämien nicht nur nach der Gefährdungsklasse richten, sondern auch individuelle sowie kollektive Präventionsmaßnahmen einpreisen. Aus ökonomischer Perspektive gibt es vier entscheidende Vorteile von risikoäquivalenten Prämien: Durch die Orientierung an den individuellen Risiken wird dem erwarteten Schaden bereits heute ein Preis gegeben. Der Preis wirkt als Signal und kann zu einem größeren Risikobewusstsein führen (Zweifel und Eisen, 2003). Es entstehen individuelle Anreize, Präventionsmaßnahmen vorzunehmen, um die eigene Risikoschadensklasse zu verbessern und eine Prämienreduktion zu erhalten. Ferner werden riskante Lagen in der langfristigen Planung teurer und damit unattraktiver. Beim Neubau werden Anreize gesetzt, die Risiken von Elementarschäden bei der individuellen Bauplanung sowie bei der Standortwahl zu berücksichtigen. Durch 
differenzierte Beiträge kann außerdem der Wettbewerb zwischen Gebietskörperschaften gefördert werden. ${ }^{8}$ Regionen, die es schaffen durch entsprechende Investitionen und geeignete Maßnahmen die Risiken von Extremwetterereignissen zu reduzieren, werden langfristig attraktiver. Letztlich kann durch risikoäquivalente Prämien eine Quersubventionierung zwischen Versicherungsnehmer:innen mit unterschiedlichen Risiken, die als ungerecht empfunden werden und damit die Zustimmungsfähigkeit einer Versicherungspflicht in der Gesellschaft verringern könnte, verhindert oder zumindest in Maßen gehalten werden.

Ein Einwand gegen risikoäquivalente Prämien ist hingegen im Umkehrschluss, dass die Versicherungsprämien für Hauseigentümer:innen in Hochrisikogebieten unter Umständen sehr hoch sein können und wie eine Enteignung wirken. Um zu gewährleisten, dass sich tatsächlich alle Gebäudeeigentümer:innen eine Elementarversicherung leisten können, sind zwei Ansätze denkbar: Zum einen könnten die Versicherungsprämien durch eine festgelegte Obergrenze gedeckelt werden. Dann ist allerdings davon auszugehen, dass der Versicherer ein Stück weit von der Risikoäquivalenz abweicht und hohe Risiken zum Teil durch niedrigere Risiken quersubventioniert werden. Soll das vermieden werden, könnte über staatliche Rückversicherungsvehikel oder Übernahmen von Ausfallgarantien für den Fall außerordentlicher Schäden nachgedacht werden. ${ }^{9}$ Zum anderen könnten Hauseigentümer:innen in Hochrisikogebieten durch Subventionen unterstützt werden. Damit dadurch nicht die positive Lenkungswirkung von risikoadäquaten Prämien zunichtegemacht wird, sollten Subventionen für die Nachfrageseite als Übergangslösung nur temporär gewährleistet werden. Sie könnten beispielsweise über einen festgelegten Zeitraum sinken, wodurch Anreize bestehen bleiben würden, zukünftig in hochgefährdeten Gebieten nicht mehr zu bauen. Hohe Risiken würden so zwar (temporär) vergemeinschaftet - allerdings gilt das auch für die aktuelle Situation, in der Ad-hocHilfsgelder von Steuerzahlenden entrichtet werden.

Weitere wichtige Überlegungen

Zusätzlich muss über eine Reihe weiterer Ausgestaltungsdetails nachgedacht werden. Beispielsweise ist es bei Ge-

8 Botzen, Aerts und van den Bergh (2009) analysieren die tatsächlichen Anreizwirkungen unterschiedlicher Risikoschadensklassen. Sie haben untersucht, inwieweit Gebäudeigentümer:innen in den Niederlanden in Präventionsmaßnahmen investieren würden, um Vergünstigungen auf ihre Versicherungsprämie zu erhalten. Danach sind zwei Drittel aller Befragten, die von Hochwasserrisiken betroffen sind, zum Zwecke einer Prämienreduktion dazu bereit, in Wasserbarrieren zu investieren.

9 Wie hoch die Versicherungsprämien für eine obligatorische Naturgefahrenabsicherung bei risikoäquivalenten Prämien für Haushalte in Deutschland sein könnten, haben Gross, Schwarze und Wagner (2019) kalkuliert. Danach liegt die monatliche Prämienhöhe für ein StandardEinfamilienhaus zwischen 5 und 50 Euro, abhängig von der Gefährdungsklasse und unter der Annahme verschiedener Selbstbehalte. bäudeversicherungen üblich, dass die Absicherung an einen Selbstbehalt geknüpft ist. Hierdurch sollen Versicherungsleistungen im Falle von Frequenzschäden - häufig vorkommende, im Ausmaß allerdings nur geringe Gebäudeschäden - verhindert werden. Einerseits können auf diese Weise Verwaltungskosten für die Versicherer, welche die Prämien ansteigen lassen würden, vermieden und positive Anreize für private Präventionsmaßnahmen gesetzt werden. Andererseits könnte es passieren, dass Versicherer durch hohe Selbstbehalte den Kontrahierungszwang umgehen oder die Prämiendeckelung kompensieren bzw. für Versicherte durch hohe Selbstbehalte die Versicherungspflicht aufgeweicht wird. Aus diesem Grund sollte auch über eine Regulierung der zulässigen Selbstbeteiligungen diskutiert werden. In eine ähnliche Richtung geht der Vorschlag nach einer Basisversicherung mit begrenzter Deckung, bei der Elementarschäden nur bis zu einer existenzsichernden Höhe kompensiert werden (Osberghaus und Wambach, 2021). Durch eine Anpassung der Sozialleistungen, wie dem Wohngeld, könnten außerdem unerwünschte Verteilungseffekte einer Versicherungspflicht zumindest für diejenigen, die eine zusätzliche Abgabe besonders hart treffen würde, abgefedert werden. Solche Maßnahmen können die allgemeine Zustimmungsfähigkeit einer Versicherungspflicht in der Gesellschaft erhöhen. Bei einer Versicherungspflicht mit mehreren Versicherungsanbietern sind zudem maximal zulässige Vertragslaufzeiten und Kündigungsfristen festzulegen. Längere Vertragslaufzeiten haben den Vorteil, dass Versicherer mehr Anreize haben, langfristig wirksame Präventionsmaßnahmen durchzusetzen. Andererseits sind Hauseigentümer:innen durch lange Vertragslaufzeiten für einen langen Zeitraum an eine Versicherung gebunden, die dadurch wiederum weniger dynamische Innovationsanreize hat.

\section{Fazit und Ausblick}

Nach den vergangenen Flutkatastrophen besteht im öffentlichen Diskurs überwiegend Einigkeit darin, dass die finanzielle Absicherung von Wohngebäuden in ihrer derzeitigen Form den erwarteten Schäden von Naturgefahren nicht gerecht wird. Die Rufe nach einer verpflichtenden Elementarschadenversicherung werden immer lauter und die zugrunde liegenden Argumente sind nicht von der Hand zu weisen. Den häufig vorgebrachten Kritikpunkten, dass eine verpflichtende Versicherung sowohl staatliche als auch individuelle Fehlanreize zur Risikovorsorge setzen würde und dass sich die Finanzierbarkeit für Gebäudeeigentümer:innen in besonders exponierten Lagen als problematisch darstellen wird, liegen konkrete Ausgestaltungsannahmen zugrunde. Diese Argumente sprechen daher nicht pauschal gegen die Einführung einer obligatorischen Naturgefahrenversicherung. Bei der Ausgestaltung muss allerdings von staatlicher Seite darauf geachtet werden, dass einerseits der Kontrahierungszwang nicht durch 
für Hauseigentümer:innen unzumutbar hohe Prämien für hohe Risiken umgangen werden kann (z. B. durch eine Kappungsgrenze), andererseits aber hinreichender Freiraum bei der Ausgestaltung der Versicherungsverträge gewährleistet wird, um langfristig sinnvolle Anreize für private und kommunale Präventionsmaßnahmen zu schaffen.

Die Einführung einer Versicherungspflicht greift erheblich in die Privatautonomie und in die Vertragsfreiheit ein. Juristische Bedenken bezüglich der Verhältnismäßigkeit bestehen weiterhin. Es bleibt abzuwarten, wie sich die Arbeitsgruppe dieses Mal positioniert und ob anschließend politische Konsequenzen folgen. Die zähen Debatten der Vergangenheit haben gezeigt, dass es sich um ein kompliziertes Problem mit einer komplexen Gemengelage und zahlreichen verschiedenen Interessen handelt. Bei der Suche nach einer angemessenen Lösung müssen Vertreter:innen aller involvierten Interessengruppen an einen runden Tisch kommen, wobei darauf geachtet werden muss, dass der Tisch auch wirklich rund ist. Zumindest aus ökonomischer Perspektive erscheinen die zunehmenden Zweifel daran berechtigt, dass auch mildere Maßnahmen als eine obligatorische Absicherung gegen Elementarschäden erfolgversprechend sein können. Es kann allerdings davon ausgegangen werden, dass sich politische Entscheidungstragende mit dem Vorschlag einer zusätzlichen Pflichtversicherung vor allem vor dem Hintergrund anhaltender Mietpreisdebatten und dem Traum vieler Bürger:innen vom Eigenheim auch auBerhalb von Wahlkampfzeiten nicht nur Freunde machen schließlich ist das, was ökonomisch sinnvoll erscheint, nicht immer automatisch politisch opportun.

\section{Literatur}

Andor, M. A., Osberghaus, D., und Simora, M. (2020). Natural disasters and governmental aid: Is there a charity hazard?, Ecological Economics, 169, 106534.

BMU - Bundesministerium für Umwelt, Naturschutz und nukleare Sicherheit (2017), Verbesserung des Schutzes vor den Folgen von Naturgefahren, https://www.umweltministerkonferenz.de/documents/0_ top41_bmub-bericht_1522238674.pdf (27. August 2021).

Botzen, W. J. W., J. C. J. H. Aerts und J. C. J. M. van den Bergh (2009), Willingness of homeowners to mitigate climate risk through insurance, Ecological Economics, 68(8), 2265-2277.

Browne, M. J. und R. E. Hoyt (2000), The Demand for Flood Insurance: Empirical Evidence, Journal of Risk and Uncertainty, 20(3), 291-306.

dpa (2021), Nach Hochwasser: Interesse an Elementarversicherungen hoch, Süddeutsche Zeitung, https://www.sueddeutsche.de/wirtschaft/versi- cherungen-nach-hochwasser-interesse-an-elementarversicherungenhoch-dpa.urn-newsml-dpa-com-20090101-210728-99-567011 (27. August 2021).

Garrett, T. A. und R. S. Sobel (2003), The Political Economy of FEMA Disaster Payments, Economic Inquiry, 41(3), 496-509.

GDV - Gesamtverband der Deutschen Versicherungswirtschaft (2019), Staat schränkt Nothilfe ein, https://www.gdv.de/de/themen/news/ staat-schraenkt-nothilfe-ein--31872 (27. August 2021).

GDV - Gesamtverband der Deutschen Versicherungswirtschaft (2021a), Mehrheit der Gebäude in Deutschland nicht richtig gegen Naturgefahren versichert, https://www.gdv.de/de/themen/news/mehrheitder-gebaeude-in-deutschland-nicht-richtig-gegen-naturgefahrenversichert-12176 (27. August 2021).

GDV - Gesamtverband der Deutschen Versicherungswirtschaft (2021b), „ZÜRS Geo“ - Zonierungssystem für Überschwemmungsrisiko und Einschätzung von Umweltrisiken, https://www.gdv.de/de/themen/ news/-zuers-geo-zonierungssystem-fuer-ueberschwemmungsisikound-einschaetzung-von-umweltrisiken-11656 (16. Dezember 2021)

GDV - Gesamtverband der Deutschen Versicherungspflicht (2021c), Was ist eine Elementarschadenversicherung?, https://www.dieversicherer.de/versicherer/versicherungen/elementarschadenversicherung (16. Dezember 2021).

Gross, C., R. Schwarze und G. G. Wagner (2019), Maßnahmen für eine zukunftsgerechte Naturgefahren-Absicherung).

Lewis, T. und D. Nickerson (1989), Self-Insurance against Natural Disasters, Journal of Environmental Economics and Management, 16(3), 209-223.

Osberghaus, D. und A. Wambach (2021), Wege zu einem besseren Versicherungsschutz gegen Hochwasserschäden, Leibniz-Zentrum für Europäische Wirtschaftsforschung, https://www.zew.de/das-zew/ aktuelles/wege-zu-einem-besseren-versicherungsschutz-gegenhochwasserschaeden (21. November 2021).

Osberghaus, D. und H. Hinrichs (2021), The Effectiveness of a Large-Scale Flood Risk Awareness Campaign: Evidence from Two Panel Data Sets, Risk Analysis, 41(6), 944-957.

Pfeiffer, M. (2021), Studie: Klimawandel mitverantwortlich für Juli-Flut, Tagesschau, https://www.tagesschau.de/inland/studie-starkregen-101. html (27. August 2021).

Raschky, P. A. und H. Weck-Hannemann (2007), Charity hazard - A real hazard to natural disaster insurance?, Environmental Hazards, 7(4), 321-329.

Schwarze, R. (2019), Institutionenökonomischer Vergleich der Risikotransfersysteme bezüglich Elementarschäden in Europa, Studien und Gutachten im Auftrag des Sachverständigenrats für Verbraucherfragen, https://www.svr-verbraucherfragen.de/wp-content/uploads/ Schwarze-12-19.pdf (27. August 2021).

Tagesschau (2021), Mehr als 29 Milliarden Euro Schaden, https://www. tagesschau.de/inland/flutkatastrophe-107.html (21. November 2021).

Ungern-Sternberg, T. von (2002), Brauchen wir eine Zwangsversicherung gegen Elementarschäden?, Wirtschaftsdienst, 82(10), 579-581, https://www.wirtschaftsdienst.eu/inhalt/jahr/2002/heft/10/beitrag/ brauchen-wir-eine-zwangsversicherung-gegen-elementarschaeden. html (3. Dezember 2021).

Verbraucherzentrale Rheinland-Pfalz (2018), Die Versicherbarkeit von Elementarschäden in der Wohngebäudeversicherung in Rheinland-Pfalz, https://www.verbraucherzentrale-rlp.de/sites/default/ files/2021-01/Marktcheck_Elementarschadenversicherung_2020.pdf (27. August 2021).

Welzer, H. (2008), Das kommunikative Gedächtnis: Eine Theorie der Erinnerung, $\mathrm{CH}$ Beck.

Zweifel, P. und R. Eisen (2003), Versicherungsökonomie, Springer Verlag, 2.

Title: Compulsory Insurance Against Damage Caused by Natural Disasters

Abstract: Like the floods of 2002 and 2013, the consequences of the flood in July 2021 have dramatically demonstrated the need for comprehensive climate adaptation measures in Germany. In this context, the government has for the third time commissioned a working group to examine an insurance obligation against natural hazards. While the basic arguments in the debate are not new, there are still several open questions on the central design and implementation details. We draw attention to the key economic trade-offs in this debate and argue that the frequent criticisms of compulsory insurance are based on specific design assumptions and therefore do not serve as general arguments against an insurance obligation. 